\title{
THE OCEAN'S GLOBAL IRON, PHOSPHORUS AND SILICON CYCLES: INVERSE MODELLING AND NOVEL DIAGNOSTICS
}

\author{
BENOÎT PASQUIER
}

(Received 5 November 2017; first published online 28 March 2018)

2010 Mathematics subject classification: primary 37N10; secondary 35J08.

Keywords and phrases: ocean biogeochemistry, nutrient cycles, dissolved iron, regenerated phosphate.

The ocean's biological pump is crucial for the carbon balance of the climate system and the control of its three-dimensional 'plumbing' on pump efficiency needs to be quantified. The nutrient cycles driving the biological pump are limited by dissolved iron (dFe). However, the iron cycle is poorly constrained and the effects of iron source perturbations have never been quantified in a data-constrained model. In this thesis, we quantify the pathways and time scales of the biological pump, build an inverse model of the coupled phosphorus, silicon and iron cycles and explore the response of these cycles to changes in the aeolian iron supply.

We use Green-function methods to show that the Southern Ocean (SO) is where $(62 \pm 2) \%$ of regenerated phosphate $\left(\mathrm{PO}_{4}\right)$ re-emerges after a mean sequestration time of $240 \pm 60 \mathrm{yr}$. The pathways from productive regions to the $\mathrm{SO}$ contribute most to the biological pump, with a mean sequestration time of $130 \pm 70 \mathrm{yr}$. Most $\mathrm{PO}_{4}$ is carried by abyssal paths with transit times exceeding $700 \mathrm{yr}$, while around $1 / 3$ of the regenerated $\mathrm{PO}_{4}$ from the equatorial Pacific that is destined for the $\mathrm{SO}$ is carried in Antarctic intermediate water.

We use the model of the coupled nutrient cycles in inverse mode to objectively determine biogeochemical parameters by minimising the mismatch with observed nutrient and phytoplankton concentrations. We generate a family of estimates, all consistent with the observations, for a wide range of iron source strengths, themselves not constrainable by current observations. The carbon and opal exports are well constrained in magnitude and pattern. We quantify the systematics of the carbon and opal exports supported by aeolian, hydrothermal and sedimentary dFe and find that aeolian $\mathrm{dFe}$ is the most efficient for supporting production.

Thesis submitted to the University of New South Wales in March 2017; degree approved on 25 June 2017; supervisor Mark Holzer.

(C) 2018 Australian Mathematical Publishing Association Inc. 
The response to aeolian source perturbations is sensitive to the state of the iron cycle that is fitted to observations. A shutdown of the aeolian source does not completely untrap nutrients from the SO because sedimentary and hydrothermal dFe suffice to

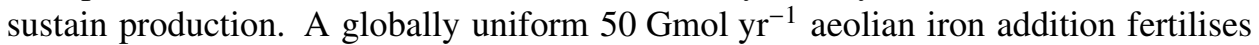
macronutrient-rich regions leading to increased deep regenerated and recycled $\mathrm{dFe}$. This perturbation actually reduces iron fertilisation supported by long-range transport because increased scavenging removes $\mathrm{dFe}$ before it can reach its destination. The response of the opal export is muted because the iron dependence of the Si:P uptake ratio counteracts fertilisation.

Parts of the thesis have been published in the papers $[1,2]$.

\section{References}

[1] B. Pasquier and M. Holzer, 'The plumbing of the global biological pump: efficiency control through leaks, pathways and time scales', J. Geophys. Res. 121(8) (2016), 6367-6388.

[2] B. Pasquier and M. Holzer, 'Inverse model estimates of the ocean's coupled phosphorus, silicon and iron cycles', Biogeosciences 14 (2017), 4125-4159.

BENOÎT PASQUIER, Department of Earth System Science, University of California, Irvine, CA, USA

e-mail: pasquieb@uci.edu 